\title{
Distance measurement system based on binocular stereo vision Jiwu Wang
}

School of Mechanical and Electronic Engineering, Beijing Jiaotong University

Beijing, Haidian District, China

Xin Pei

School of Mechanical and Electronic Engineering, Beijing Jiaotong University

Beijing, Haidian District, China

E-mail: jwwang@bjtu.edu.cn,19125998@bjtu.edu.cn

www.bjtu.edu.cn

\begin{abstract}
The distance measurement system based on binocular stereo vision is proposed. The binocular vision model is established, Matlab toolbox is used to calibrate and obtain the internal and external parameters of the binocular camera. Then stereo rectification and stereo matching are carried out with OpenCV. Finally, the depth information is obtained by the parallax measurement of image pairs, and the distance between any two points can be calculated. The simulation experiments have been done and the results demonstrated that the measuring method is feasible.
\end{abstract}

Keywords: stereo vision; camera calibration; stereo matching; distance measurement.

\section{Introduction}

Distance measurement is one of the most important tasks of a computer vision system, and it is also continues to be a popular research topic in computer and robot vision nowadays. Distance measurement plays an important role in robot navigation, obstacle detection, navigation of autonomous vehicles, surveillance monitoring, person localization and tracking, and many more. The distance measurement system based on binocular stereo vision is proposed in this paper. Firstly, the binocular vision model is established, a GUI interface is designed in MATLAB for calibration and the display of parameters. Then stereo rectification and stereo matching are carried out with OpenCV. Finally, the depth information is obtained by the parallax measurement of image pairs, and the distance between any two points can be calculated. The simulation experiments have been done and the results demonstrated that the measurement method is feasible.
The designed binocular stereo vision system consists of five modules: image acquisition, camera calibration, image rectification, stereo matching, and distance measurement. This system can be described by the flow diagram shown in Figure 1.

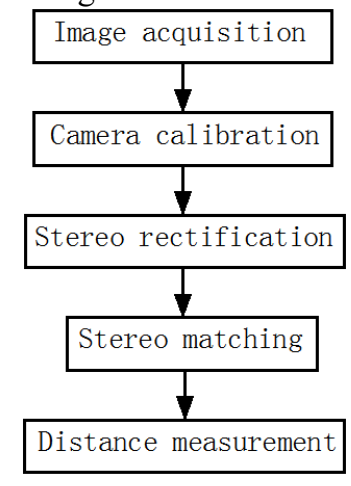

Fig. 1. System flow chart

2. Principle of binocular stereo vision ranging

(C) The 2021 International Conference on Artificial Life and Robotics (ICAROB2021), January 21 to 24, 2021 
Binocular stereo vision distance measurement is modeled on the distance perception methods of human eyes. In this paper, the stereo vision system consists of two cameras with the same parameters that is parallel mounted and looking at the same objects, as shown in Figure 2. The projection model is created based on the imaging principle of pinhole camera. The object points $\mathrm{P}$ are given in a world coordinate system. $\mathrm{P}_{1}$ and $\mathrm{P}_{\mathrm{r}}$ are imaging points on left and right images. Assuming that the image plane of the left and right cameras is located on the same plane and the two images are aligned, it can be known from the principle of similar triangle that:

$$
\frac{B-\left(x_{l}-x_{r}\right)}{Z-f}=\frac{B}{Z}
$$

Therefore, it can be derived from equation (1):

$$
Z=\frac{f B}{x_{l}-x_{r}}
$$

Where, $\mathrm{B}$ is the base distance between the two cameras, and $f$ is the focal length of the camera lens. Again, $x l$ and $\mathrm{xr}$ respectively represent the pixel distance of $\mathrm{Pl}$ and $\mathrm{Pr}$ in the $\mathrm{X}$-axis direction in their respective coordinate system. The difference in the coordinates of the corresponding pixels is known as disparity $\mathrm{d}$. $\mathrm{Z}$ is the depth distance of the object point from the camera position.

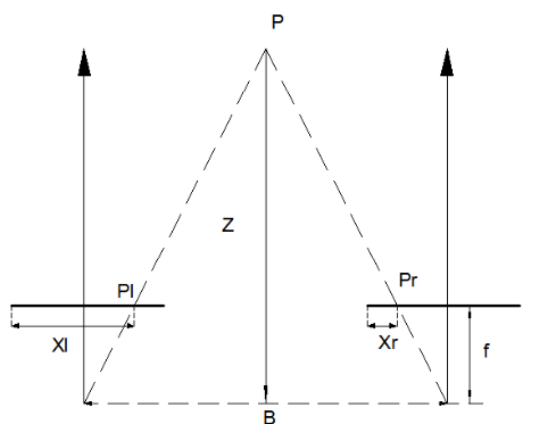

Fig. 2. Binocular vision model

\section{Camera calibration}

The process of calibration is actually the process of solving the internal and external parameters of the camera. Calibration is the basic and necessary process of binocular stereo vision. The result of calibration will determine the accuracy of distance measurement of the target object. In the process of camera calibration, three coordinate systems are needed: image coordinate system, camera coordinate system and world coordinate system. The camera is approximated to a pinhole model, and the parameter $\mathrm{S}$ (scale factor) and the homography matrix $\mathrm{H}$ are introduced. The relationship between the points $P=\left[\begin{array}{ll}X & Y\end{array}\right]^{T}$ in the imaging plane and $P=\left[\begin{array}{lll}X & Y & Z\end{array}\right]^{T}$ in the space can be expressed as: $P=s H P$

Where $\mathrm{H}$ is represented by two matrices: $H=M W$

$$
M=\left[\begin{array}{ccc}
f_{x} & 0 & c_{x} \\
0 & f_{y} & c_{y} \\
0 & 0 & 1
\end{array}\right]
$$

$\mathrm{M}$ is the intrinsic parameter matrix of the camera. Where, $c_{x}$ and $c_{y}$ represent coordinates in pixel of the image center respectively. Since pixels on a normal camera are not guaranteed to be square, use two different focal lengths $\mathrm{f}_{\mathrm{x}}$ and $\mathrm{f}_{\mathrm{y}}$.

$W=[R T]$,where, $\mathrm{R}, \mathrm{T}$ are the extrinsic parameters of the cameras, denoting the rotation matrix and the translation vector from the left camera coordinate system to the right camera coordinate system, respectively.

Considering the distortion of lens, suppose $q_{p}\left(x_{p}, y_{p}\right)$ is the corrected point, and $q_{p}\left(x_{p}, y_{p}\right)$ is the distorted point, then:

$$
\left[\begin{array}{l}
x_{p} \\
y_{p}
\end{array}\right]=\left(1+k_{1} r^{2}+k_{2} r^{4}+k_{3} r^{6}\right)\left[\begin{array}{l}
x_{d} \\
y_{d}
\end{array}\right]+\left[\begin{array}{l}
2 p_{1} x_{d} y_{d}+p_{2}\left(r^{2}+2 x_{d}^{2}\right) \\
p_{1}\left(r^{2}+2 y_{d}^{2}\right)+2 p_{2} x_{d} y_{d}
\end{array}\right]
$$

Where $\left(\mathrm{K}_{1}, \mathrm{~K}_{2}, \mathrm{P}_{1}, \mathrm{P}_{2}, \mathrm{~K}_{3}\right)$ constitutes a $5 \times 1$ matrix, which is the distortion matrix of the camera.

The method of camera calibration used in this paper is based on the checkerboard template calibration method proposed by Zhang, which has the advantages of simple operation and high precision. In this paper, a GUI interface is designed in MATLAB for calibration and display of results. The specific steps are as follows:

(i) Self-made calibration plate; The number of c corners is $7 \times 5$ and the size of the calibration target is $20 \mathrm{~mm} \times 20 \mathrm{~mm}$

(ii) Image acquisition; Images from different angles of the calibration target are collected simultaneously with a binocular camera placed in parallel, and the image resolution is $640 \times 480$. A total of 13 sets of image pairs were collected, as shown in Figure 3 and Figure 4. 


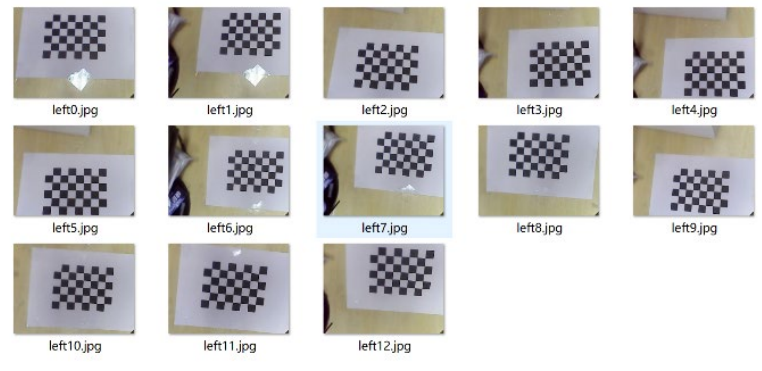

Fig. 3. Left images of calibration target images

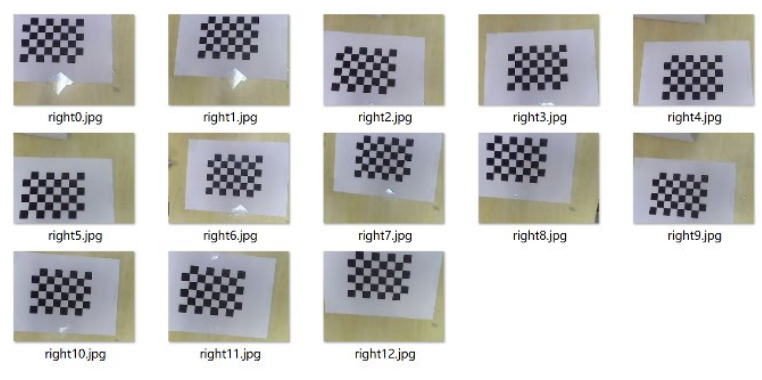

Fig. 4. Right images of calibration target images

(iii) Calibration. Click the calibration button on the GUI interface to import the binocular image pair collected. Then, corner detection and stereo calibration are carried out to obtain the internal and external parameters of the binocular camera and display them on the GUI interface, as shown in Figure 5.

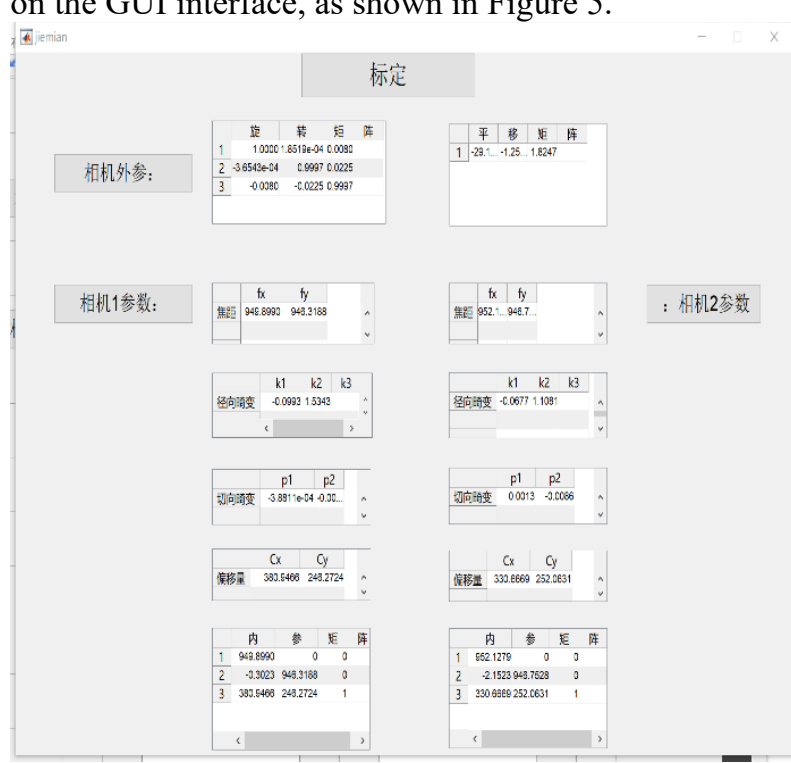

Fig. 5. GUI interface

\section{Stereo rectification}

The purposes of image rectification are as follows: the distortions of images are removed by the internal parameters in the calibration results, and the two images aligned in non-coplanar lines are rectified to coplanar line alignment such that the polar lines of the two images lie exactly on the same horizontal line. Any point on such an image must have the same line number as its corresponding point on another image. Therefore, in case of searching for corresponding points in two images, it is only necessary to search in the same epipolar line, reducing a $2 \mathrm{D}$ search space to $1 \mathrm{D}$ to reduce the search time effectively.

The stereo rectification can be realized by the function of cvStereoRectify in OpenCV. Figure 6 shows the left and right image pairs before stereo rectification. Figure 7 shows the image pairs after stereo rectification.

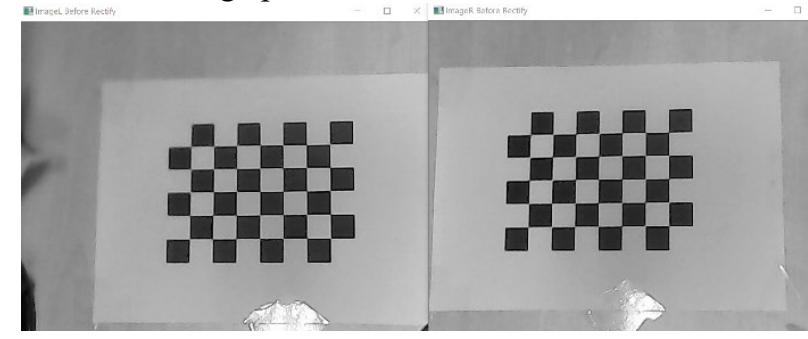

Fig. 6. image pairs before stereo rectification

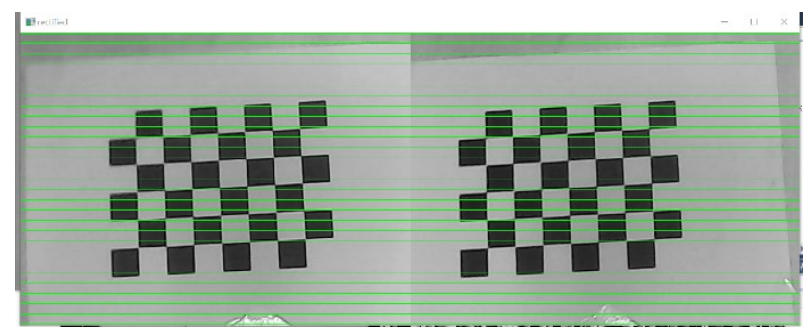

Fig. 7. image pairs after stereo rectification

\section{Stereo matching}

The stereo matching algorithm is an important step in generating disparity maps. Whether stereo matching is accurate directly affects the accuracy of binocular ranging results. The basic principle of stereo matching is to find the corresponding relationship between the pixels of the same scene in the projected image under different viewpoints, and then to obtain the distance information of object by calculating the parallax between the pixels.

(C) The 2021 International Conference on Artificial Life and Robotics (ICAROB2021), January 21 to 24, 2021 
In this paper, CV Find Stereo Correspondence BM, a block-matching Stereo Correspondence algorithm provided by Open $\mathrm{CV}$, is used to find the matching points between the left and right images after stereo rectification through a small window (SAD) called " Sum of Absolute Difference ".

Through matching the left and right rectified image pairs in OpenCV, the disparity map can be obtained. In the disparity map, any two target points can be selected with the mouse to obtain their depth information, and the distance between the two points can be calculated, as shown in Figure 8.

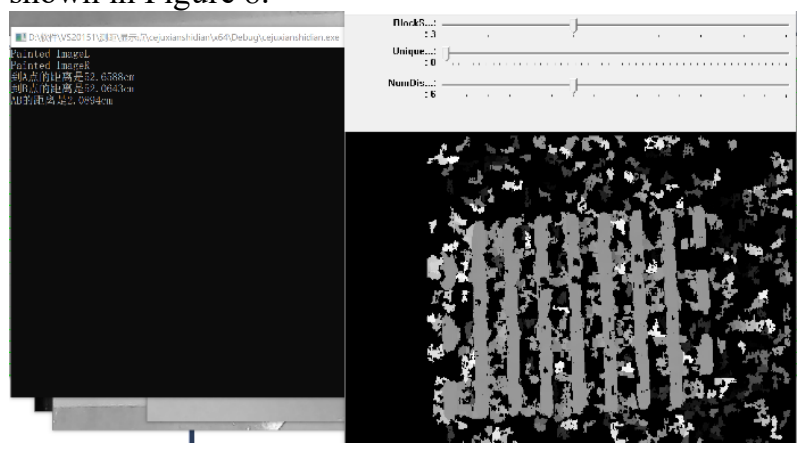

Fig. 5. Disparity map

The actual distance between the corners of the calibration plate is $20 \mathrm{~mm}$. From the results of experiments, it can be seen that the measurement method basically meets the requirement of precision, the measuring method is feasible.

\section{Conclusion}

A procedure for distance measurement using a binocular stereo vision system was presented in this article. The experimental results demonstrated that the system could meet basically the needs of measuring. The measurement method is feasible for applications where accurate distance measurement is not required and could offer better technical support for machine vision applications. However, due to the influence of matching and other factors, the accuracy should be further improved in the future research work.

\section{Acknowledgements}

This work was supported by the Key Research and Development Program (No.M18GY300021).

\section{References}

1. Gary Bradski, Adrian Kaebler. Learning OpenCV[M]. Translated by Yu Shiqi and Liu Ruizhen. Beijing: Tsinghua University Press, 2009.

2. Hu Hanping. Research on key Technologies of Binocular stereoscopic ranging [D]. Graduate University of The Chinese Academy of Sciences (Changchun Institute of Optics, Fine Mechanics and Physics),2014.

3. Zhao Xunjie, Li Chengjin. Research on key Technologies of binocular Stereo Real-time ranging System [J]. Laser \& Infrared,2006(09):874-877.

4. Wang Zhenzhen, Zhao Lianyu, Liu Zhenzhong. Binocular stereo ranging system based on MATLAB and OpenCV $[\mathrm{J}]$. Journal of tianjin university of technology,2013,29(01):45-48.

5. Shen Tong, Liu Wenbo, WANG Jing. Target ranging system based on binocular stereo vision $[\mathrm{J}]$. Electronic measurement technology,2015,38(04):52-54.

6. Wang Hao, Xu Zhiwen, Xie Kun, Li Jie, Song Chenglu. Binocular ranging system based on OpenCV [J]. Journal of jilin university (information science),2014,32(02):188194.

\section{Authors Introduction}
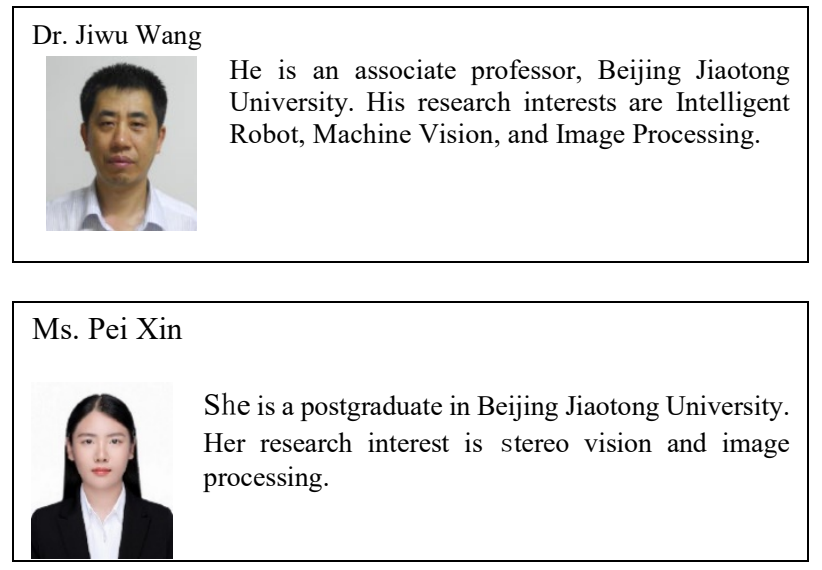